\title{
(C) OPEN ACCESS \\ Changes in smoking cessation assistance in the European Union between 2012 and 2017: pharmacotherapy versus counselling versus e-cigarettes
}

\author{
Filippos T Filippidis, ${ }^{1,2}$ Anthony A Laverty, ${ }^{1}$ Ute Mons, ${ }^{3}$ Carlos Jimenez-Ruiz, ${ }^{4}$ \\ Constantine I Vardavas ${ }^{2,5,6}$
}

${ }^{1}$ Public Health Policy Evaluation Unit, School of Public Health, Imperial College London, London, UK

${ }^{2}$ Center for Health Services Research, School of Medicine, National and Kapodistrian University of Athens, Athens, Greece

${ }^{3}$ Cancer Prevention Unit and WHO Collaborating Centre for Tobacco Control, German Cancer Research Center, Heidelberg, Germany ${ }^{4}$ Smoking Cessation Service, Community of Madrid, Madrid, Spain

5 Institute of Public Health, American College of Greece, Athens, Greece

${ }^{6}$ Nicotine Dependence Center, Mayo Clinic, Rochester, Minnesota, USA

Correspondence to Dr Filippos T Filippidis, Department of Primary Care and Public Health, School of Public Health, Imperial College London, London W6 8RP, UK: f.filippidis@imperial.ac.uk

Received 25 October 2017 Revised 25 February 2018 Accepted 26 February 2018 Published Online First 21 March 2018

\section{ABSTRACT}

Background The landscape of smoking cessation may have changed in Europe recently.

Objectives To identify changes in use of smoking cessation assistance in the European Union (EU) and factors associated with use of cessation assistance. Methods Data from the $2012(n=9921)$ and 2017 ( $n=9489$ ) waves of the Eurobarometer survey were used. Self-reported use of smoking cessation assistance was assessed among smokers who had ever tried to quit and former smokers. Changes in use of each type of assistance were assessed using logistic regression. Results Among current and former smokers, those who had ever attempted to quit without assistance increased from $70.3 \%$ (2012) to $74.8 \%$ (2017). Current smokers were more likely to have used any assistance compared with former smokers $(P<0.001)$. Use of e-cigarettes for smoking cessation assistance increased $(3.7 \%$ to $9.7 \%) \%$ ), while use of pharmacotherapy $(14.6 \%$ to $11.1 \%) \%$ ) and smoking cessation services (7.5\% to $5.0 \%) \%$ ) declined. Younger people were more likely to have reported e-cigarette use for smoking cessation but less likely to have used a cessation service. Individuals living in countries with comprehensive smoking cessation policies were more likely to have used any cessation assistance (adjusted OR (aOR) $=1.78 ; 95 \% \mathrm{Cl} 1.15$ to 2.76), pharmacotherapy (aOR=3.44; $95 \% \mathrm{Cl} 1.78$ to 6.66 ) and smoking cessation services ( $\mathrm{aOR}=2.27$; $95 \% \mathrm{Cl} 1.27$ to 4.06 ) compared with those living in countries with weak smoking cessation policies.

Conclusions These findings highlight the need for approaches that ensure that smokers get support to quit smoking across the EU. The question of whether the availability of e-cigarettes will displace other methods, and the impact of such a displacement, should be closely evaluated.

\section{INTRODUCTION}

The WHO has selected a $30 \%$ reduction in tobacco use as one of the 25 by 2025 goals, and the WHO Regional Office for Europe has professed their ultimate goal to have a European region free of tobacco use. ${ }^{1}$ Smoking cessation is thus a core activity for population health and entails substantial health benefits both for individual smokers and public health. Smoking cessation has additionally been recognised as an essential component of the WHO's MPOWER package for tobacco control and the WHO Framework Convention for Tobacco Control (FCTC). ${ }^{2}$

There is, however, wide variation between EU Member States (EU MS) in both their smoking prevalence and in the extent of their tobacco control policies. A 2014 review identified gaps in the implementation of the FCTC in Europe, including highlighting concerns that the region was progressing slowly in terms of providing comprehensive cessation services-an important aspect of FCTC Article 14. ${ }^{3}$ A previous analysis of Eurobarometer data across the EU found that the majority of smokers who were trying to quit tobacco use were not using assistance. Moreover, the use of cessation assistance varies among countries, depending in part on their policies regarding access to cessation methods. ${ }^{4}$ This variation in provision of cessation assistance has raised concerns over how people are attempting to quit, especially if they are unable to access evidencebased smoking cessation methods. This is particularly important for clinicians dealing with patients trying to quit with a variety of methods for which they may have little experience or limited evidence.

In light of this gap, we conducted secondary analyses of pooled Eurobarometer datasets from 2012 and 2017 in order to assess the changing landscape of smoking cessation assistance used across EU MS during the past 5 years among current and former smokers. As a secondary outcome, we also investigated potential EU MS-specific changes in the use of cessation assistance and sociodemographic factors associated with the use of particular types of assistance.

\section{METHODS}

\section{Data source}

We analysed data from 27 EU MS, collected in two Eurobarometer surveys: wave 77.1 (February-March 2012) and wave 87.1 (March 2017)..$^{5}$ All Eurobarometer surveys employ a multistage sampling design in each MS according to which primary sampling units (PSUs) are selected from each region within each country, proportional to population size. Subsequently, a sample of starting addresses is randomly selected in each PSU, and households are systematically selected following a standard random route starting from these initial addresses. One participant in each household is randomly selected and interviewed in the local language. The methodology has been 
consistent across Eurobarometer waves. The European Commission does not publish response rates; however, poststratification and population size weighting were applied in each country/ region to produce nationally representative samples in terms of age, gender and area of residence. A total of $n=26751$ and $n=26853$ individuals aged $\geq 15$ years from 27 EU MS were interviewed in 2012 and 2017, respectively. A total of 19410 respondents (9921 in 2012 and 9489 in 2017) were included in the analysis as either former smokers or current smokers who reported a previous quit attempt. Interviews in Croatia, the 28th EU MS, were only conducted in 2017; therefore, it was excluded from this analysis.

\section{Measures}

\section{Tobacco smoking}

All respondents were asked 'Regarding smoking cigarettes, cigars or a pipe, which of the following applies to you?'. Responses included 'You currently smoke' (ie, current smokers); 'You used to smoke but you have stopped' (ie, former smokers); and 'You have never smoked' (ie, never smokers).

\section{Use of cessation assistance}

Former smokers, as well as current smokers who had ever tried to quit smoking in the past were asked: 'Which of the following did you use in order to quit or to try to quit smoking?'. Respondents could choose one or more responses. Wording of some responses differed slightly between the two surveys, but for the purpose of this analysis, we grouped them as follows: (A) medication, including nicotine replacement medications (nicotine gum, patch, inhaler and so on (nicotine replacement therapy (NRT))) or other medications ('Pharmacotherapy'); (B) health services, including support from the doctor or other health professional or special stop-smoking services (clinics, specialists, quitlines and so on) ('Smoking cessation services'); (C) oral tobacco (snus), chewing or nasal tobacco (snuff) ('Oral or nasal tobacco'); (D) electronic cigarettes or similar devices ('Electronic cigarettes'); (E) other; and (f) you quit or you tried to quit without assistance ('Without assistance'). Respondents who mentioned at least one of options (A) to (E) were classified as having used any smoking cessation assistance in the past.

\section{Sociodemographic data}

Respondents also provided data on their age (15-24, 25-39, $40-54$ and $\geq 55$ years), sex (male; female), age at which they stopped full-time education ( $\leq 15,16-19$ and $\geq 20$ years old), their difficulties to pay bills during the last 12 months (almost never/never and from time to time/most of the time) and area of residence (rural and urban).

\section{National cessation policies}

We collected data on national smoking cessation policies from the Tobacco Control Scale (TCS) $2013^{7}$ and 2016, ${ }^{8}$ which score European countries according to their tobacco control policies. We used the score for the subscale 'treatment to help smokers stop', which can range from 1 to 10 points and evaluates recording of smoking status in medical notes (1 point); brief advice in primary care (1 point); quitline ( 2 points); network of smoking cessation support and its reimbursement (4 points); and reimbursement of medications (2 points). We classified member states into three categories: low (1-4), medium (5-7) and high TCS treatment score (8-10). Country-specific scores varied very little between 2013 and 2016; therefore, the classification for each member state was consistent over time. Ireland was the only country that could be potentially classified into two categories ( 7 points in 2013 and 8 points in 2016). We used the most recent score to classify it as a high-scoring country.

\section{Statistical analysis}

We restricted our analysis to former smokers and current smokers who reported having tried to quit in the past. Descriptive results are presented as \% with 95\% CIs and regression results as adjusted ORs with 95\% CI. Proportions are compared with $\chi^{2}$ tests. Multilevel logistic regression models with country as the higher level of analysis were fitted to assess the association between independent variables (age, sex, education, difficulty paying bills, area of residence, TCS treatment score and year of survey) and each of the following outcomes: (A) having used any cessation assistance; (B) having tried to quit without assistance; (C) having used pharmacotherapy; (D) having used smoking cessation services; and (E) having used electronic cigarettes or similar devices. The multilevel model controls for clustering within countries. An interaction term between TCS treatment score and calendar year was included to explore whether change in use of cessation assistance differed according to comprehensiveness of smoking cessation policies. Associations with the remaining cessation types of assistance were not assessed, as the number of responses was too low to be able to draw any reliable results. Separate models, adjusting for the same variables, were run for each EU MS to assess differences between 2012 and 2017 in the reported use of the three most commonly reported types of assistance: pharmacotherapy, smoking cessation services and electronic cigarettes. Finally, correlation coefficients between country level changes (ie, change in ever use between 2012 and 2017 as a percentage of the 2012 value) in ever use of pharmacotherapy, smoking cessation services and e-cigarettes were calculated. All analyses were performed with Stata V.14.0, and weights provided in the Eurobarometer dataset were used in descriptive analyses to account for the complex sampling design of the survey.

\section{RESULTS}

Among current and former smokers, the majority of respondents had tried to quit without assistance both in 2012 (70.3\%) and in 2017 (74.8\%) (table 1). Pharmacotherapy was the most popular cessation assistance (14.6\% in 2012 and $11.1 \%$ in 2017), followed by electronic cigarettes and cessation services. More current smokers who had attempted to quit reported having used any cessation assistance compared with former smokers both in 2012 (40.4\% vs 25.6\%, P<0.001) and in $2017(37.5 \%$ vs $20.8 \%, \mathrm{P}<0.001)$. Among those who had ever used any assistance to quit smoking, $16.6 \%$ of respondents in 2012 and $12.1 \%$ in $2017(\mathrm{P}<0.001)$ reported having ever used more than one type of assistance. Including 'without assistance' as a cessation method, $9.5 \%$ of our sample in 2012 and $6.0 \%$ of our sample in 2017 reported having used multiple methods to quit $(\mathrm{P}<0.001)$. In $2017,35.3 \%$ of those who had ever used pharmacotherapy, $34.9 \%$ of those who had ever used cessation services, $32.1 \%$ of those who had ever used e-cigarettes to quit and only $4.1 \%$ of those who had ever tried without assistance reported having tried to quit with multiple methods.

Former smokers and current smokers who had tried to quit were less likely to have used any cessation assistance in 2017 , compared with $2012(\mathrm{aOR}=0.61 ; 95 \% \mathrm{CI} 0.52$ to 0.71$)$ and consequently more likely to have tried quitting without any assistance $(\mathrm{aOR}=1.72 ; 95 \% \mathrm{CI} 1.47$ to 2.02$)$, but these changes were attenuated in countries with medium and high TCS treatment 
Table 1 Use of smoking cessation assistance among former smokers and current smokers who have tried to quit in the European Union, 20122017

\begin{tabular}{|c|c|c|c|c|c|c|}
\hline & \multicolumn{2}{|c|}{$\begin{array}{l}\text { Current smokers who had ever tried to quit } \\
\%(95 \% \mathrm{Cl})\end{array}$} & \multicolumn{2}{|l|}{$\begin{array}{l}\text { Former smokers } \\
\%(95 \% \mathrm{Cl})\end{array}$} & \multicolumn{2}{|l|}{$\begin{array}{l}\text { Total } \\
\%(95 \% \mathrm{Cl})\end{array}$} \\
\hline & $\begin{array}{l}2012 \\
n=4341\end{array}$ & $\begin{array}{l}2017 \\
n=3489\end{array}$ & $\begin{array}{l}2012 \\
n=5580\end{array}$ & $\begin{array}{l}2017 \\
n=6000\end{array}$ & $\begin{array}{l}2012 \\
n=9921\end{array}$ & $\begin{array}{l}2017 \\
n=9489\end{array}$ \\
\hline Pharmacotherapy & 22.5 (20.5 to 24.5$)$ & 16.6 (14.6 to 18.8$)$ & 8.5 (7.5 to 9.7$)$ & 7.5 (6.5 to 8.7) & 14.6 (13.6 to 15.8$)$ & $11.1(10.1$ to 12.3$)$ \\
\hline $\begin{array}{l}\text { Smoking cessation } \\
\text { services }\end{array}$ & 8.5 (7.3 to 9.9$)$ & 5.7 (4.5 to 7.1$)$ & 6.7 (5.8 to 7.8 ) & 4.5 (3.7 to 5.4 ) & 7.5 (6.7 to 8.4$)$ & 5.0 (4.3 to 5.8$)$ \\
\hline Electronic cigarettes & 7.1 (5.9 to 8.4) & 15.6 (13.5 to 17.8$)$ & $1.1(0.7$ to 1.5$)$ & 5.8 (4.8 to 6.9$)$ & 3.7 (3.2 to 4.3$)$ & 9.7 (8.7 to 10.8 ) \\
\hline Oral or nasal tobacco & $0.6(0.4$ to 0.9$)$ & $1.1(0.7$ to 1.9$)$ & $0.7(0.6$ to 1.1$)$ & 1.2 (0.9 to 1.6$)$ & $0.7(0.5$ to 0.9$)$ & $1.2(0.8$ to 1.5$)$ \\
\hline Without assistance & 65.7 (63.4 to 67.9) & 66.2 (63.4 to 68.8 ) & 73.9 (72.1 to 75.7$)$ & 80.7 (78.9 to 82.3$)$ & 70.3 (68.9 to 71.7 ) & 74.8 (73.3 to 76.3 ) \\
\hline Any assistance & 40.4 (38.1 to 42.7 ) & 37.5 (34.8 to 40.3$)$ & 25.6 (23.8 to 27.4$)$ & 20.8 (19.2 to 22.6$)$ & 32.2 (30.8 to 33.7$)$ & 27.5 (26.0 to 29.1$)$ \\
\hline
\end{tabular}

scores as shown by the interaction terms of TCS treatment score and year (table 2). The reported use of e-cigarettes as cessation assistance was almost two times higher in countries with low TCS treatment score in the 2017 wave $(\mathrm{aOR}=1.85 ; 95 \%$ CI 1.31 to 2.61) and much higher in countries with medium and high TCS treatment scores. In contrast, respondents were less likely to report having used pharmacotherapy $(\mathrm{aOR}=0.73 ; 95 \% \mathrm{CI}$ 0.58 to 0.93 ) and health professional/cessation service advice
$(\mathrm{aOR}=0.53 ; 95 \% \mathrm{CI} 0.38$ to 0.74$)$ in 2017 when compared with 2012. The effect size for use of health professional/cessation services did not differ among countries with different TCS treatment scores.

People living in countries with more comprehensive smoking cessation policies (ie, with high TCS treatment scores) were more likely to have used any cessation assistance $(\mathrm{aOR}=1.78 ; 95 \% \mathrm{CI}$ 1.15 to 2.76 ), pharmacotherapy (NRT or other medication)

Table 2 Associations of self-reported smoking cessation assistance with sociodemographic factors and tobacco treatment policies in 27 EU MS among former smokers and current smokers who had tried to quit, 2012-2017 ( $n=19410)$

\begin{tabular}{|c|c|c|c|c|c|}
\hline & $\begin{array}{l}\text { Any assistance* aOR } \\
(95 \% \mathrm{Cl})\end{array}$ & $\begin{array}{l}\text { Without assistance aOR } \\
(95 \% \mathrm{Cl})\end{array}$ & $\begin{array}{l}\text { Pharmacotherapy aOR } \\
(95 \% \mathrm{Cl})\end{array}$ & $\begin{array}{l}\text { Health services aOR } \\
(95 \% \mathrm{Cl})\end{array}$ & $\begin{array}{l}\text { Electronic cigarettes aOR } \\
(95 \% \mathrm{Cl})\end{array}$ \\
\hline \multicolumn{6}{|l|}{ Year } \\
\hline 2012 (ref) & 1.00 & 1.00 & 1.00 & 1.00 & 1.00 \\
\hline 2017 & 0.61 (0.52 to 0.71$)$ & 1.72 (1.47 to 2.02 ) & 0.73 (0.58 to 0.93 ) & 0.53 (0.38 to 0.74$)$ & 1.85 (1.31 to 2.61$)$ \\
\hline \multicolumn{6}{|c|}{ Tobacco Control Scale treatment score } \\
\hline Low (ref) & 1.00 & 1.00 & 1.00 & 1.00 & 1.00 \\
\hline Medium & 1.20 (0.86 to 1.68$)$ & 0.92 (0.68 to 1.26$)$ & 1.59 (0.95 to 2.65$)$ & 1.47 (0.93 to 2.31$)$ & $1.19(0.72$ to 1.97$)$ \\
\hline High & 1.78 (1.15 to 2.76$)$ & 0.57 (0.38 to 0.86$)$ & 3.44 (1.78 to 6.66$)$ & 2.27 (1.27 to 4.06$)$ & 1.58 (0.83 to 3.01$)$ \\
\hline \multicolumn{6}{|c|}{ Year*Tobacco Control Scale treatment score } \\
\hline $2017^{*}$ Low (ref) & 1.00 & 1.00 & 1.00 & 1.00 & 1.00 \\
\hline $2017 *$ Medium & 1.39 (1.17 to 1.66$)$ & 0.64 (0.54 to 0.77$)$ & 1.17 (0.90 to 1.51$)$ & $1.22(0.85$ to 1.77$)$ & $1.28(0.87$ to 1.89$)$ \\
\hline $2017 *$ High & $1.43(1.14$ to 1.78$)$ & $0.64(0.51$ to 0.80$)$ & 0.69 (0.50 to 0.93$)$ & 0.81 (0.51 to 1.28$)$ & 2.42 (1.53 to 3.82$)$ \\
\hline \multicolumn{6}{|l|}{ Age (years) } \\
\hline$\geq 55$ (ref.) & 1.00 & 1.00 & 1.00 & 1.00 & 1.00 \\
\hline $40-54$ & 1.45 (1.34 to 1.58$)$ & 0.73 (0.67 to 0.79$)$ & 1.69 (1.52 to 1.88$)$ & 0.91 (0.78 to 1.06$)$ & 2.32 (1.94 to 2.77$)$ \\
\hline $25-39$ & 1.32 (1.21 to 1.45$)$ & 0.81 (0.73 to 0.88 ) & 1.33 (1.18 to 1.50$)$ & 0.66 (0.55 to 0.79 ) & 2.89 (2.40 to 3.47$)$ \\
\hline $15-24$ & $1.38(1.20$ to 1.60$)$ & 0.80 (0.69 to 0.92 ) & 0.88 (0.71 to 1.10$)$ & 0.54 (0.38 to 0.74 ) & 4.38 (3.43 to 5.60$)$ \\
\hline \multicolumn{6}{|l|}{ Sex } \\
\hline Female (ref.) & 1.00 & 1.00 & 1.00 & 1.00 & 1.00 \\
\hline Male & 0.98 (0.92 to 1.05$)$ & $1.03(0.96$ to 1.10$)$ & 0.89 (0.82 to 0.98$)$ & 1.00 (0.88 to 1.13$)$ & 1.06 (0.93 to 1.21$)$ \\
\hline \multicolumn{6}{|l|}{ Difficulties paying bills } \\
\hline Never/almostnever (ref.) & 1.00 & 1.00 & 1.00 & 1.00 & 1.00 \\
\hline $\begin{array}{l}\text { From time to time/most of } \\
\text { the time }\end{array}$ & 1.19 (1.10 to 1.28$)$ & 0.84 (0.78 to 0.91 ) & 1.22 (1.11 to 1.35$)$ & 1.29 (1.12 to 1.49$)$ & 1.35 (1.17 to 1.57$)$ \\
\hline \multicolumn{6}{|c|}{ Age when stopped education (years) } \\
\hline Up to 15 (ref.) & 1.00 & 1.00 & 1.00 & 1.00 & 1.00 \\
\hline $16-19$ & 1.18 (1.06 to 1.31$)$ & 0.88 (0.79 to 0.98 ) & 1.33 (1.15 to 1.54$)$ & 1.21 (1.00 to 1.46$)$ & 1.30 (1.03 to 1.63$)$ \\
\hline$\geq 20$ & $1.12(1.01$ to 1.25$)$ & 0.93 (0.83 to 1.04$)$ & 1.24 (1.06 to 1.44$)$ & 1.26 (1.03 to 1.55$)$ & 1.15 (0.90 to 1.46$)$ \\
\hline \multicolumn{6}{|l|}{ Area of residence } \\
\hline Rural (ref.) & 1.00 & 1.00 & 1.00 & 1.00 & 1.00 \\
\hline Urban & 1.07 (1.00 to 1.15$)$ & 0.94 (0.88 to 1.01$)$ & 1.04 (0.95 to 1.15$)$ & 1.12 (0.98 to 1.28 ) & 1.13 (0.97 to 1.31$)$ \\
\hline
\end{tabular}

*Includes pharmacotherapy, smoking cessation services, electronic cigarettes, oral or nasal tobacco and other.

aOR, adjusted OR; EU MS, EU Member States. 
Pharmacotherapy

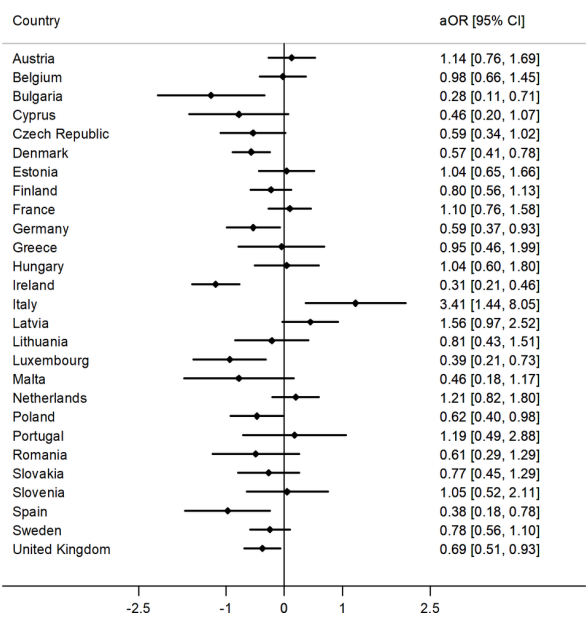

Cessation Services

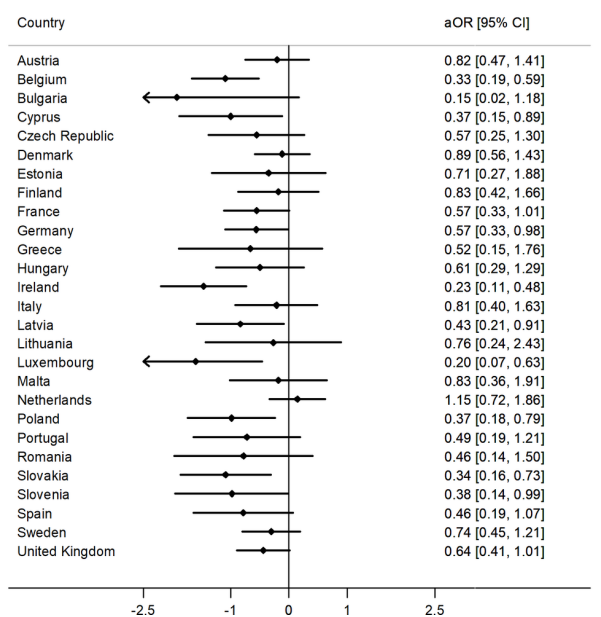

E-cigarettes

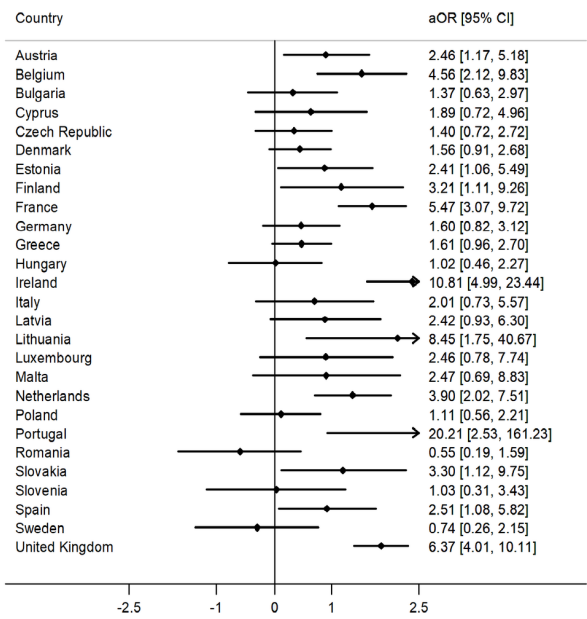

Figure 1 Changes (aOR with 95\% Cl) between 2012 and 2017 in having used pharmacotherapy, cessation services and e-cigarettes, respectively, as cessation assistance among former smokers and current smokers who had tried to quit in $27 \mathrm{EU}$ member states $(\mathrm{n}=19410)$. ${ }^{*}$ Cessation services include support from the doctor or other health professional or special smoking cessation services (clinics, specialists, quitlines and so on). Pharmacotherapy includes nicotine replacement medications (nicotine gum, patch, inhaler and so on) or other medications. aOR, adjusted OR; EU, European Union.

$(\mathrm{aOR}=3.44 ; 95 \% \mathrm{CI} 1.78$ to 6.66$)$ and health professionals/ smoking cessation services $(\mathrm{aOR}=2.27$; 95\% CI 1.27 to 4.06$)$ compared with those living in countries with low TCS treatment scores. There was no statistically significant difference regarding the use of e-cigarettes.

Younger people (15-24 years) were more likely than those aged $\geq 55$ years to have used any cessation assistance including e-cigarettes (aOR 4.38 ; $95 \%$ CI 3.43 to 5.60 ) but less likely to have received advice from health professionals/smoking cessation services (aOR $0.54 ; 95 \%$ CI 0.38 to 0.74 ). People with difficulties paying bills were more likely to have used any cessation assistance than those who never or almost never have problems paying their bills. Sex, with the exception of pharmacotherapy, and area of residence were not significantly associated with the use of cessation assistance.

Variations in changes between 2012 and 2017 across EU MS are shown in figure 1 . The reported past use of pharmacotherapy was less likely in eight of the 27 EU MS in 2017, compared with 2012 and more likely only in Italy, while the remaining 18 MS experienced non-statistically significant changes. A statistically significant increase in the use of electronic cigarettes to quit smoking was reported in 12 EU MS. In nine EU MS, support from health professionals/smoking cessation services was less likely to be reported in 2017, compared with 2012, with no statistically significant increase in any of the EU MS (figure 1). Changes in pharmacotherapy and cessation services use at the MS level were modestly correlated $(\mathrm{r}=0.53)$. On the contrary, change in e-cigarette use was weakly correlated with both changes in pharmacotherapy $(r=0.22)$ and in cessation services $(r=0.18)$.

\section{DISCUSSION}

This secondary analysis of two recent Eurobarometer waves found that the use of any self-reported smoking cessation assistance decreased in the EU between 2012 and 2017. Self-reported use of e-cigarettes for smoking cessation increased, while the use of standard cessation assistance, such as pharmacotherapy and support from healthcare professionals and cessation services, was less likely to be reported in 2017 compared with 2012 . There was considerable variation between EU MS, with changes differing depending on the level of tobacco treatment policies. Notably, in countries with more comprehensive smoking cessation policies, adults reported substantially higher use of pharmacotherapy and cessation services, indicating the impact of national policies on cessation attempts.

The increase in the reported use of electronic cigarettes as cessation assistance coinciding with a decrease in the use of pharmacotherapy or support from health professionals is consistent with findings from the English Smoking Toolkit study conducted between 2011 and late 2014. ${ }^{9}$ That study concluded that there was no evidence that the decline in the use of NRT could be attributed to the rise in the use of e-cigarettes, a finding they confirmed also using more recent data. ${ }^{10}$ Use of medication and NRT for smoking cessation can be influenced by factors such as the availability of new pharmaceuticals, funding and smoke-free legislation ${ }^{11-14}$; therefore, the explanation of these changes may differ between EU MS. In our study, it is not clear whether using e-cigarettes as a substitute for tobacco impacts use of established services like NRT and smoking cessation clinics or whether it is mostly employed by smokers who would otherwise try to quit without assistance, especially due to the inexistence of a supportive policy environment for standard cessation assistance (pharmacotherapy and cessation services). At the individual MS level, there was only weak correlation between changes in e-cigarette use and changes in use of standard cessation assistance, which might suggest coincidence rather than displacement. More research on whether e-cigarette use is displacing standard cessation assistance in Europe and how this may impact longterm abstinence is required, especially considering the increase in e-cigarette use. ${ }^{15} 16$

While changes in cessation assistance and support used were quite similar in the majority of EU MS, we nevertheless observed some heterogeneity between countries. As a previous international study of differences in cessation assistance in 15 countries noted, such variation may reflect a combination of differences in tobacco control efforts and priorities, the affordability of different methods and more general cultural factors. ${ }^{17}$ In our analyses, national policies regarding availability and cost of cessation services were associated with both use of the assessed 
cessation assistance and changes over time. Our findings are in line with an earlier analysis of Eurobarometer data from 2012, which found that living in a country that offers cost-covered national quitlines, medication and other cessation services was associated with higher likelihood of using standard cessation assistance with proven efficacy. ${ }^{4}$ Although there seems to be an overall decline in use of cessation assistance for smoking cessation across the $\mathrm{EU}$, this concerning trend was attenuated in MS with more comprehensive cessation services, highlighting the importance of national policies and FCTC Article 14 implementation. It has to be noted, however, that the question used for the analysis refers to 'ever use of cessation assistance', so it would take a considerable amount of time to detect an effect of any policy change, as there is already a substantial number of people who had used certain cessation assistance in quit attempts before the current policies were put into place. This limitation of our analysis further highlights the increase in e-cigarette use for cessation, as it is a product that has become available much more recently compared with other types of cessation assistance, although respondents may have been more likely to recall assistance used in more recent quit attempts.

Our results have important implications for clinical practice, as they highlight the fact that European healthcare professionals are increasingly having to deal with smokers who are using e-cigarettes for smoking cessation and are less likely to use well established cessation assistance. ${ }^{18-21}$ Regardless of factors related to access and cost of cessation services, our findings may also reflect the reluctance of some smokers to engage in 'medicalised' cessation that involves contact with health services or use of medication. Beyond questions surrounding the efficacy of e-cigarettes in smoking cessation, ${ }^{22}$ for which there is limited evidence from randomised controlled trials, ${ }^{23}$ this trend poses a new challenge for health professionals, whose experience in handling smoking cessation among smokers who use e-cigarettes or wish to use e-cigarettes is still limited. This challenge also extends to regulating bodies, which should closely monitor research on novel products that are or may be used in quit attempts and consider updating their guidance to clinicians accordingly.

It is also important to explore country-specific factors that may have influenced the use of smoking cessation assistance in recent years and could explain the variability in changes between member states. In the UK, for example, increase in e-cigarette use was coupled with a decline in use of pharmacotherapy and cessation services, although these opposing trends may not be associated. ${ }^{91014}$ On the contrary, despite an increase in e-cigarette use for smoking cessation in the Netherlands, use of pharmacotherapy and cessation services has not changed, while other member states, such as Germany and Poland, experienced decreased use of pharmacotherapy and cessation services without a concurrent change in the use of e-cigarettes over the study period. It is also interesting to note that no statistically significant changes were reported in Greece, despite worsening access to healthcare services overall during the economic crisis. ${ }^{24}$ These examples underscore the complexity of the smoking cessation landscape in the EU and the multitude of factors that may shape future developments in each member state.

In our analysis, we found that a number of smokers have tried to quit smoking using multiple methods, including a variety of cessation assistance and trying without assistance. This is consistent with findings from the USA, ${ }^{25}$ although the difference in the cessation assistance assessed does not allow direct comparisons. Moreover, our data did not allow us to explore whether different types of cessation assistance were used concurrently or in distinct quit attempts. In addition, those who successfully quit reported much lower use of cessation assistance compared with smokers who had tried to quit without success. This is likely explained by the fact that smokers who find it more difficult to quit are also more likely to seek assistance; therefore, our study cannot provide insight into the relative effectiveness of smoking cessation methods.

\section{Strengths and limitations}

We used two cross-sectional surveys with large samples representative of the EU population and consistent sampling methodology; therefore, we are confident that these conclusions are generalisable to the entire EU population, although the samples at the country level were modest, typically around 1000 individuals in each member state. The samples were independently selected in the two waves (no cohort was followed up), hence any causal interpretations of the detected associations should be made with caution. Further cohort study data across the EU is needed. ${ }^{26}$ The classification of policies regarding cessation services may also not provide adequate granularity to capture small but potentially important variations in policies between EU MS. Additionally, we had no data regarding the time when each type of cessation assistance had been used, which would have helped us identify changes specifically associated with recent use of cessation assistance, nor was any information on efficacy available. All data were self-reported and thus may be subject to bias, as people may be more likely to remember cessation assistance used more recently or, in the case of former smokers, those that were effective in helping them to quit. Former smokers were defined as such based on self-reports; no minimum period of abstinence was reported. Finally, we combined data for cigarettes, cigars and pipes; however, because regular use of cigars and pipes is rare in the $\mathrm{EU},{ }^{27}$ any effect on our results is most likely minimal.

\section{CONCLUSION}

The majority of attempts to quit smoking in the EU continue to be without any cessation assistance. The proportion of smokers attempting to quit using assistance has further decreased over the past 5 years. The use of established aids such as pharmacotherapy have become less popular, while e-cigarettes as a potential cessation or switching method has grown between 2012 and 2017. This highlights the need to evaluate their efficacy and impact on individual abstinence as well as their population-level implications and to explore whether they may be displacing standard cessation assistance. Finally, it is important to emphasise the central role of the smoking cessation policy environment. Living

What this paper adds

- Use of e-cigarettes for smoking cessation has increased, while use of pharmacotherapy and smoking cessation services has decreased across the European Union between 2012 and 2017.

- Changes differed widely among member states. Individuals living in countries with comprehensive smoking cessation policies were more likely to have used any cessation assistance.

- Smoking cessation is a key strategy in the battle against tobacco both at a population and at the individual levels. Identifying trends and factors associated with the use of cessation assistance can inform both policy makers and clinicians. 
in a country that offers cost-covered national quitlines, pharmacotherapy and other cessation services was associated with higher likelihood of using standard cessation assistance with proven efficacy, highlighting the importance of national policies in FCTC Article 14 implementation.

Acknowledgements The Public Health Policy Evaluation Unit at Imperial College is grateful for the support of the NIHR School of Public Health Research.

Contributors FTF had the main role in study conception and data analysis. All authors contributed to manuscript preparation and data interpretation. All authors have read and approved the final version of the manuscript.

Funding This work was supported by a grant from the European Commission (Horizon2020 HCO-6- 2015; EUREST-PLUS: 681109; Vardavas). AAL receives funding from the NIHR (RP 2014-04-032).

Competing interests None declared.

Patient consent Detail has been removed from this case description/these case descriptions to ensure anonymity. The editors and reviewers have seen the detailed information available and are satisfied that the information backs up the case the authors are making.

Ethics approval Data were freely available and deidentified, thus no ethical approval was required.

Provenance and peer review Not commissioned; externally peer reviewed.

Data sharing statement The datasets supporting the conclusions of this article are freely available online, doi:10.4232/1.12265 in www.gesis.org.

Open access This is an open access article distributed in accordance with the Creative Commons Attribution Non Commercial (CC BY-NC 4.0) license, which permits others to distribute, remix, adapt, build upon this work non-commercially, and license their derivative works on different terms, provided the original work is properly cited and the use is non-commercial. See: http://creativecommons.org/ licenses/by-nc/4.0/

C Article author(s) (or their employer(s) unless otherwise stated in the text of the article) 2019. All rights reserved. No commercial use is permitted unless otherwise expressly granted.

\section{REFERENCES}

1 World Health Organization - Regional Office for Europe. Roadmap of actions to strengthen implementation of the WHO Framework Convention on Tobacco Control in the European Region 2015-2025: making tobacco a thing of the past, 2015.

2 World Health Organization. WHO report on the global tobacco epidemic, 2008: The MPOWER package. Geneva, 2008.

3 World Health Organization. European tobacco control status report 2014: WHO Regional Office for Europe, 2014.

4 Filippidis FT, Gerovasili V, Vardavas $\mathrm{Cl}$, et al. Determinants of use of smoking cessation aids in 27 European countries. Prev Med 2014;65:99-102.

5 European Commission. Eurobarometer 77.1, February-March 2012. GESIS Data Archive: ZA5597, dataset version 2.0.0 (2012). Brussels: TNS OPINION \& SOCIAL, 2012.
6 European Commission. Eurobarometer 87.1, March 2017. GESIS Data Archive: ZA6861, Data file Version 1.2.0,10.4232/1.12922. Brussels: TNS OPINION \& SOCIAL, 2017.

7 Joossens L, Raw M. The tobacco control scale 2013 in Europe, 2013.

8 Joossens L, Raw M. The tobacco control scale 2016 in Europe, 2017.

9 Beard E, Brown J, McNeill A, et al. Has growth in electronic cigarette use by smokers been responsible for the decline in use of licensed nicotine products? Findings from repeated cross-sectional surveys. Thorax 2015;70:974-8.

10 Beard E, West R, Michie S, et al. Association between electronic cigarette use and changes in quit attempts, success of quit attempts, use of smoking cessation pharmacotherapy, and use of stop smoking services in England: time series analysis of population trends. BMJ 2016;354:i4645.

11 Szatkowski L, Coleman T, McNeill A, et al. The impact of the introduction of smokefree legislation on prescribing of stop-smoking medications in England. Addiction 2011;106:1827-34.

12 Jarlenski M, Hyon Baik S, Zhang Y. Trends in use of medications for smoking cessation in medicare, 2007-2012. Am J Prev Med 2016;51:301-8.

13 Kasza KA, Cummings KM, Carpenter MJ, et al. Use of stop-smoking medications in the United States before and after the introduction of varenicline. Addiction 2015;110:346-55.

14 Britton J. Electronic cigarettes and smoking cessation in England. BMJ 2016;354:14819.

15 Filippidis FT, Laverty AA, Gerovasili V, et al. Two-year trends and predictors of e-cigarette use in 27 European Union member states. Tob Control 2017;26.

16 Filippidis FT, Laverty AA, Vardavas Cl. Experimentation with e-cigarettes as a smoking cessation aid: a cross-sectional study in 28 European Union member states. BMJ Open 2016;6: 012084.

17 Borland R, Li L, Driezen P, et al. Cessation assistance reported by smokers in 15 countries participating in the International Tobacco Control (ITC) policy evaluation surveys. Addiction 2012;107:197-205.

18 Cahill K, Stevens S, Perera R, et al. Pharmacological interventions for smoking cessation: an overview and network meta-analysis. Cochrane Database Syst Rev 2013:5:CD009329.

19 Stead LF, Perera R, Bullen C, et al. Nicotine replacement therapy for smoking cessation. Cochrane Database Syst Rev 2012;11:CD000146.

20 Stead LF, Buitrago D, Preciado N, et al. Physician advice for smoking cessation. Cochrane Database Syst Rev 2013;5:CD000165.

21 West R, Raw M, McNeill A, et al. Health-care interventions to promote and assist tobacco cessation: a review of efficacy, effectiveness and affordability for use in national guideline development. Addiction 2015;110:1388-403.

22 Kalkhoran S, Glantz SA. E-cigarettes and smoking cessation in real-world and clinical settings: a systematic review and meta-analysis. Lancet Respir Med 2016;4:116-28.

23 Hartmann-Boyce J, McRobbie H, Bullen C, et al. Electronic cigarettes for smoking cessation. Cochrane Database Syst Rev 2016;9:CD010216.

24 Filippidis FT, Gerovasili V, Millett C, et al. Medium-term impact of the economic crisis on mortality, health-related behaviours and access to healthcare in Greece. Sci Rep 2017;7:46423.

25 Caraballo RS, Shafer PR, Patel D, et al. Quit methods used by us adult cigarette smokers, 2014-2016. Prev Chronic Dis 2017;14:E32.

26 Vardavas C. EUREST PLUS - european regulatory science on tobacco: policy implementation to reduce lung diseases - proposal (Horizon2020). Tob Prev Cessat 2016;2.

27 European Commission. Special Eurobarometer 458 "Attitudes of Europeans towards tobacco and electronic cigarettes". Brussels, 2017. 